\title{
EATING DISORDERS IN ADOLESCENTS - PHYSICAL AND PSYCHIATRIC PERSPECTIVES
}

Dr Rajeev Ramachandran, A/Prof John Wong Chee Meng

\section{ABSTRACT}

Eating disorder (ED) referrals of school age children and adolescents, by their parents and school teachers, have become more common. Also, they are now presenting at an earlier age to the primary health care and school systems, with physical, medical and psychological symptoms. Nevertheless, there is an average of six months to two years between the onset of symptoms to formal assessment and treatment by specialist team. There are also more cases presenting to ED specialist clinic services, especially pre-pubertal children, with early onset and presentation before 14 years old. Mid and late adolescent presentations (after 14 years old) continue to make up more than two third of the cases. More than 60 percent of cases seen in specialist clinics are of the restrictive type anorexia nervosa, and often associated with persistent and excessive exercise. Thirty percent of cases presented are Bulimia nervosa, which tend to be episodic. Majority of single episode bulimia cases do not present themselves early to medical services but take on open source self-directed management. For patients with bulimia who comply to treatment program and recover after 6-12 months of therapy, they can also experience high relapse rate as they often discontinue their follow up. Avoidant-restrictive food intake disorder is more closely related to pre-pubertal onset eating disorder with arrested sexual maturity and growth failure, if left untreated. Psychiatric co-morbidities arising from body image disturbance, overdrive high achievement needs, prior exposure to adverse childhood experiences (ACE), dysfunctional family or peer relationships, include anxiety, avoidance behavior, obsessive rumination, depression, suicidal ideation and attempt. Death can arise from acute presentation and chronic state of ED, when associated with medical complications from refeeding syndrome, severe malnourishment, accidents and suicide. Early identification and assessment by family physicians would significantly improve the prognosis and mitigate against long term chronicity when share care with ED specialist services.

Keywords: eating disorder, anorexia, bulimia, ARFID, BMI, weight, body image, BMI, refeeding, food anxiety, obsessional rumination, depression, suicide

SFP2021; 47(4): 24-27

Rajeev Ramachandran

Head Adolescent Medicine and Senior Consultant, $\mathrm{NUH}$

John Wong Chee Meng

Head Psychological Medicine Senior Consultant, NUH and NUS

\section{INTRODUCTION}

Eating disorders (EDs) are primarily psychiatric illnesses with significant, life-threatening medical complications. The risk of premature death is 6-12 times higher in females with Anorexia Nervosa (AN) as compared to the general population. ${ }^{1}$ Anorexia nervosa has the highest mortality rate of any psychiatric disorders. Medical complications account for more than half of all deaths in patients with anorexia nervosa and rest is due to suicide. $^{2}$

Early diagnosis and intervention improve the recovery and outcomes in eating disorders. ${ }^{3}$ General practitioners and family physicians well informed in the early detection, presentation and management of eating disorders, could improve the prognosis and clinical outcome of the patient.

Evidence-based, multidisciplinary care approach is the standard of care. Members of the multidisciplinary team generally consists of psychiatrist, physician, psychologist and dietitian. Family Based Therapy (FBT) and Cognitive Behavioral Therapy (CBT) are considered to be most effective method of treatment for Anorexia Nervosa in children and young adults. Family physicians trained in Eating Disorder could also provide early assessment, monitoring and supportive care of adolescent ED patients and their families, and mitigate against the risk of recurrence when in remission.

\section{EATING DISORDERS}

The common eating disorders include Anorexia Nervosa, Bulimia Nervosa, Avoidance/Restrictive Food Intake Disorder (ARFID) and Binge-Eating Disorder.

1. Anorexia Nervosa (AN): Patients with AN see themselves as overweight, even when they are dangerously underweight. Due to the intense fear of gaining weight, they severely restrict their food intake, often exercise excessively, and/or may force themselves to vomit or use laxatives to lose weight. Anorexia Nervosa has the highest mortality rate of any mental disorder. The death could be due to medical complications associated with malnutrition or refeeding, some may die of suicide.

2. Bulimia Nervosa (BN): Patients with $\mathrm{BN}$ have recurrent and frequent episodes of eating unusually large amounts of food and feeling a lack of control over these episodes. This binge-eating is followed by compensatory behaviors like forced vomiting, excessive use of laxatives or diuretics, fasting, excessive exercise, or a combination of these behaviors. 
3. Avoidant/Restrictive Food Intake Disorder (ARFID): These patients experience disturbed eating either due to a lack of interest in eating or a distaste for certain smells, tastes, colors, textures or temperatures. They may develop weight loss and nutritional deficiency but without weight or shape concerns.

4. Binge Eating Disorder (BED): Binge eating without compensatory behavior is the feature of this condition. Patients with BED often are overweight or obese. Binge-eating disorder is probably the most common eating disorder in the community but they are not commonly seen in hospitals.

\section{PRESENTING SIGNS AND SYMPTOMS}

Patients with EDs specifically AN may often come to hospitalwith medical complications from the disease. In addition to the cognitive and behavioral problems that characterize the EDs, the following physical signs and symptoms can occur in patients with an ED as a consequence of restricting food or fluid intake, nutritional deficiencies, binge-eating, and inappropriate compensatory behaviors, such as purging. It is important to know that lot of these patients may not have any symptoms or signs.

They may present to any general physician with non-specific symptoms like weight loss, cold intolerance, weakness/fatigue or lethargy, dizziness, fainting etc. They may see an endocrinologist or $\mathrm{O} \& \mathrm{G}$ doctor for amenorrhea or oligomenorrhea and infertility. They may go to a psychiatrist for depressive, anxiety, obsessive-compulsive symptoms and behaviors, memory loss, attention issues, insomnia or self-harm behaviors. They may visit a gastroenterologist for abdominal bloating, epigastric pain, GE reflux symptoms, constipation etc. Dermatologists may see them for lanugo hair (fine hair growth on the body and face), hair loss, carotenemia (yellowish discoloration of skin), Russell's sign (calluses or scars on the back of the hand from self-induced vomiting), dry brittle hair and nails. Some of them might get referred to cardiologists for chest pain, palpitations, bradycardia and hypotension.

ED is associated with several medical complications. ${ }^{4}$ Almost all major organ systems gets affected. Some organs are more affected than others. Certain organs get affected earlier than others. The primary risk factors for developing medical complications in anorexia nervosa are the degree of weight loss and the chronicity of the illness. Surprisingly, patients with anorexia nervosa do not seem to be predisposed to frequent infectious diseases, notwithstanding their malnourished states. They tend to recover faster from infections as well.
Table 1. Medical complications of anorexia nervosa

\begin{tabular}{|l|l|}
\hline Cardiovascular & Endocrine and Metabolic \\
\hline Bradycardia and hypotension & Amenorrhea \\
\hline Mitral valve prolapse & Infertility \\
\hline Arrhythmia & Osteoporosis \\
\hline Pericardial effusion & Sick euthyroid \\
\hline Dermatologic & Hypoglycemia \\
\hline Dry skin & Hematologic \\
\hline Alopecia & Pancytopenia \\
\hline Lanugo hair & Neurologic \\
\hline Gastrointestinal & Cerebral atrophy \\
\hline Constipation & Attention deficit \\
\hline Hepatitis & Pulmonary \\
\hline Dysphagia & Aspiration pneumonia \\
\hline $\begin{array}{l}\text { Superior Mesenteric Artery } \\
\text { (SMA) syndrome }\end{array}$ & Spontaneous pneumothorax \\
\hline
\end{tabular}

\section{INITIAL ASSESSMENT}

The initial assessment of individuals with eating disorders involves a thorough review of the patient's history (including psychosocial - HEADSSS), current symptoms, physical condition, weight control measures, and co-morbid psychiatric issues or disorders such as depression, anxiety, substance abuse, or personality issues. The initial assessment is the first step in establishing a diagnosis and treatment plan.

COMPLETE HISTORY - We should find out the rate and amount of weight loss in the past few months.Current eating patterns should be probed. Find out about purging behaviors, and exercise patterns. Dietitian should be involved to get a detailed nutritional assessment. Nutritional history should include dietary intake (quantity and variety of foods consumed), restriction of specific foods or food groups (such as fats or carbohydrates). Compensatory behaviors and their frequency (fasting or dieting, induced vomiting, laxative, diuretic abuse, use of diet pills, alternative medications and other over-the-counter slimming medicines). Menstrual history - age of menarche, last menstrual period and regularity should be documented. Family history including symptoms or diagnosis of EDs, obesity, mood \& anxiety disorders, substance abuse. Psychiatrist and psychologist should be involved in getting a detailed psychological assessment.

\section{Assessment Tools}

Screening of high risk individuals in primary care can be useful with instrument like SCOFF ${ }^{8}$, followed by a clinical consultation. See Table 2.

\section{Table 2. SCOFF Screening questions}

- Do you make yourself Sick because you feel uncomfortably full?

- Do you worry you have lost Control over how much you eat?

- Have you recently lost more than One stone $(6.35 \mathrm{Kg})$ in a 3-month period?

- Do you believe yourself to be Fat when others say you are too thin? - Would you say that Food dominates your life?

One point for every yes,; a score of $=/>2$ indicates a likely cause of anorexia or bulimia 
Standardized self-report questionnaires like Eating Attitude Test -26 items $^{9}$ (EAT-26) provides an objective measure of symptoms and concerns of eating disorder, and is highly reliable and valid. It has also been used as a screening tool in schools, college among at risk youth population. The Eating Disorder Examination Questionnaires (EDE-Q) ${ }^{10}$ also provides a self-report measurement of symptom response to treatment progression.

\section{PHYSICAL EXAMINATION}

Physical Examination should include measurement of height, weight, and body mass index (BMI) and plot them on growth charts for children and adolescents. Check vital signs- identify bradycardia, hypotension and orthostatic changes. Look for skin, hair and nail changes - lanugo hair, dental caries, oral ulcers, Russell's sign and evidence of self-harm.

\section{INITIAL DIAGNOSTIC EVALUATION}

$\mathrm{AN}$ is the most common ED that will present to the hospital with medical instability. Laboratory and other diagnostic studies need to be performed to screen for potential abnormalities seen in patients with $\mathrm{AN}$. It is important to note that initial laboratory studies may be normal even with significant malnutrition. The body's compensatory mechanisms are remarkable and laboratory abnormalities may not be observed until the illness if far advanced. ${ }^{2}$

Initial lab tests include FBC, ESR, Renal Panel-2, Mg, Liver Function Test, Thyroid Screen, FSH, LH and oestradiol. ECG and BMD (whole body) should be obtained.

Besides EAT-26, psychological rating scales could include Beck Youth Inventory- Second Edition (BYI for 7 to 18 years old) to profile domains in depression, anxiety, anger, disruptive behavior and self-concept.

\section{PSYCHIATRIC CO-MORBIDITIES}

AN may present with obsessions and compulsive behaviors and a strong drive for perfection in personal goals and achievements, besides obsessional ruminations about eating habits and keeping weight low. Rigid personality traits often make the treatment compliance challenging. Depression is a common early presentation and associated with low self-esteem, failures to achieve self-determined goals. Anxiety especially in anxiety when presented with food, and social anxiety are upsetting for the patients.

BN which could run a more chronic course, and may have transited from AN. It is associated with obsessive compulsive disorder, depression, body dysmorphic disorder.

ARFID tends to be associated with anxiety symptoms, less with depression compared to AN and BN. Some may be associated with autistic spectrum disorder, cognitive impairment and intellectual disability.

Guilt and shame, self-harm, suicidal ideation, substance abuse, impulsive risk taking behavior, in the background of mood lability and dysregulations are common ${ }^{11}$.

\section{TREATMENT}

Outpatient care is effective for mild cases of AN in early phase of presentation, if medically stable and psychiatric risk is managed. Medically unstable patients would need in patient stabilization. ED services provide comprehensive treatment programs covering outpatient and residential meal supervision, intensive psychological treatment treating underlying psychiatric conditions.

\section{INDICATIONS FOR REFERRAL AND HOSPITALIZATION FOR ACUTE MEDICAL STABILIZATION}

Presence of one or more of the following -

1. Heart rate of less than 50 beats per minute $(<45 \mathrm{bpm}$ during sleep),

2. Systolic blood pressure below $90 \mathrm{~mm} \mathrm{Hg}$,

3. A postural blood pressure drop of greater than $10 \mathrm{~mm}$ of $\mathrm{Hg}$, increase in HR > 20bpm,

4. The presence of cardiac arrhythmias,

5. A temperature below $35.5^{\circ} \mathrm{C}$ (hypothermia),

6. Electrolyte abnormalities - hypo/hypernatremia, hypophosphatemia, hypokalemia,

7. Suicidal ideation,

8. Refusal to eat or ongoing weight loss despite intensive outpatient treatment,

9. Body weight less than 75 percent of ideal body weight.

\section{TREATMENT}

Refeeding syndrome describes the clinical and metabolic derangements that can occur during refeeding of a malnourished patient. The clinical features of refeeding syndrome include edema, cardiac and/or respiratory failure, gastrointestinal problems, profound muscle weakness, delirium and, in extreme cases, death. The most important biochemical change is hypophosphatemia. Other expected problems are hypoglycemia, hypokalemia, hypomagnesemia and hyponatremia. Refeeding syndrome is a potentially fatal condition which requires specialized care in an inpatient unit. ${ }^{5}$ The risk of developing refeeding syndrome is higher if the degree of malnutrition at presentation is severe, in chronic malnutrition and with rapid or profound weight loss.

Management of refeeding syndrome - Patient should be admitted to hospital and closely monitored. Serum electrolytes (sodium, potassium, phosphorous and magnesium) and glucose should be checked prior to initiating supervised feeding. They may be normal prior to refeeding.

Phosphorus will reach its lowest point three to seven days after initiation of nutritional rehabilitation. Keep monitoring the serum electrolytes and glucose frequently (daily if significantly abnormal) during early refeeding until they get stabilized. Aggressively replete all electrolyte deficiencies. Oral repletion is safer. It is not necessary to correct fluid and electrolyte imbalance before initiating feeding. Start a multivitamin daily and add thiamine supplementation in severely malnourished patients.

Closely monitor vital signs during refeeding period.With the help of dietitian, adjust amount of calorie intake. The previously 
practiced "Start low and go slow" methods of refeeding have recently been changed to a more rapid refeeding with close medical monitoring. This is now preferred during inpatient treatment as it is found to be safe and reduces the duration of hospital stay. ${ }^{6}$ The objective is to achieve $1-1.5 \mathrm{~kg}$ weight gain every week during the weight restoration phase in the hospital.

Patient may be discharged once medical stabilization is achieved. Outpatient therapy will mostly focus on psychological issues and further weight gain will help in recovery of the body organ functions. The full resolution of symptoms may take an extended period of treatment. Full recovery from AN is achieved when weight restoration has been successfully completed, there is absence of distorted body image and other abnormal eating behaviors, psychological comorbidities and any social or functional impairments

\section{PSYCHIATRIC TREATMENT}

Many early cases of adolescents with ED are now embarking on open source web resources on self-help treatment. There are promising evidence on the effectiveness of self-help intervention when adhered to. However, cases with complex psychological comorbidities and failure to adhere to treatment often requires detail assessment and supervised structured treatment plan by the multi-disciplinary team once diagnosed.

Cognitive behavioral therapy (CBT) is evidence based as first line treatment for both bulimia nervosa and binge eating disorder. CBT is also effective for treatment of AN, while family based therapy (FBT) with parent coaching are evidenced based in the treatment of younger adolescents with $\mathrm{AN}$, where parental support and supervision are critical to engage these younger teens.

Medication can be useful adjunct to nutritional and psychological treatments for ED, targeting at treating underlying co-morbid psychiatric disorder of depression, anxiety, obsessional rumination, and improving weight gain. Recent evidence support modest weight restoration benefits from Olanzapine. SSRI antidepressant e.g. Fluoxetine at high dose (up to $40-60 \mathrm{mg}$ when tolerated), may be useful in reducing bingeing and purging episodes and overall $\mathrm{BN}$ severity ${ }^{12}$.

\section{REFERENCES}

1.Löwe B, Zipfel S, Buchholz C, Dupont Y, Reas DL, Herzog W. Long-term outcome of anorexia nervosa in a prospective 21-year follow-up study. Psychological medicine. 2001 Jul;31(5):881-90.

2. National Institute for Clinical Excellence. Eating disorders: Core interventions in the treatment and management of anorexia nervosa, bulimia nervosa and related eating disorders. National Institute for Clinical Excellence; 2004.

3. Campbell K, Peebles R. Eating disorders in children and adolescents: state of the art review. Pediatrics. 2014 Sep 1;134(3):582-92

4. Nervosa CT. Diagnosis and care of patients with anorexia nervosa in primary care settings. Ann Intern Med. 2001;134:1048-59.

5. Academy for Eating Disorders. AED Report 2016. Eating Disorders: A Guide to Medical Care. 3rd Edition. [Internet]. 2016 [cited 27 Jun 2019]. Available from: https:// higherlogicdownload.s3.amazonaws.com/AEDWEB/05656ea0-59c9-4dd4-b83207a3fea58f4c/UploadedImages/AED_Medical_Care_Guidelines_English_04_03_18_a.pdf

6. Garber AK, Mauldin K, Michihata N, Buckelew SM, Shafer MA, Moscicki AB. Higher calorie diets increase rate of weight gain and shorten hospital stay in hospitalized adolescents with anorexia nervosa. Journal of Adolescent Health. 2013 Nov 1;53(5):579-84.

7. Lee HY, Lee EL, Pathy P, Chan YH. Anorexia nervosa in Singapore: an eight-year retrospective study. Singapore medical journal. 2005 Jun;46(6):275.

8. Morgan JF, Reid F, Lacey JH. The SCOFF questionnaire: assessment of a new screening tool for eating disorders. Bmj. 1999 Dec 4;319(7223):1467-8.

9. Garner DM, Olmsted MP, Bohr Y, Garfinkel PE. The eating attitudes test: psychometric features and clinical correlates. Psychological medicine. 1982 Nov;12(4):871-8.

10. Mond JM, Hay PJ, Rodgers B, Owen C, Beumont PJ. Validity of the Eating Disorder Examination Questionnaire (EDE-Q) in screening for eating disorders in community samples. Behaviour research and therapy. 2004 May 1;42(5):551-67.

11. Dogra N, Lunn B, Cooper S, editors. Psychiatry by ten teachers. CRC Press; 2017 Jan 20.

12. Mairs R, Nicholls D. Assessment and treatment of eating disorders in children and adolescents. Archives of Disease in Childhood. 2016 Dec 1;101(12):1168-75.

\section{LEARNING POINTS}

Key leaning points about eating disorders are:

- Common, under diagnosed, and access to care is often delayed.

- It can progress to become life threatening.

- Early treatment is often associated with improved outcome.

- Associated with physical health complications affecting multi-organ and systems.

- Physical assessment and psychological interview conducted in a sensitive and engaging way is critical.

- Management of anorexia nervosa is psychological based, coupled with medical management of potential risk from weight restoration.

- Management of bulimia nervosa is centered on cognitive behavioural therapy.

- Trained Family Physicians could play a key role in early detection, assessment, initiate treatment with dieticians and psychological therapy, refer and share care with ED services in follow up management of recovered cases from developing chronicity. 